\title{
The Hidden Charm Decay of $X(3872), Y(3940)$ and Final State Interaction Effects
}

\author{
Xiang Liu * Bo Zhang, and Shi-Lin Zhu \\ Department of physics, Peking University, Beijing, 100871, China
}

(Dated: June 21, 2018)

\begin{abstract}
We investigate whether the final state interaction (FSI) effect plays a significant role in the large hidden charm decay width of $\mathrm{X}(3872)$ and $\mathrm{Y}(3940)$ using a model. Our numerical result suggests (1) the FSI contribution to $X(3872) \rightarrow J / \psi \rho$ is tiny; (2) $\Gamma\left[Y(3940) \rightarrow D \bar{D}^{*}+\right.$ h.c. $\left.\rightarrow J / \psi \omega\right]$ from FSI is around several keV, far less than Belle's experimental value $7 \mathrm{MeV}$.
\end{abstract}

PACS numbers: 13.30.Eg, 13.75.Lb, 14.40.Lb

\section{INTRODUCTION}

The underlying structure of $X(3872)$ is still very controversial. It was discovered by Belle collaboration [1] and confirmed by Babar 2], CDF [3] and D0 [4] collaborations. Recently, Belle collaboration reported a new decay mode $X(3872) \rightarrow D^{0} \bar{D}^{0} \pi^{0}[5$. The mass of $X(3872)$ from various experiments reads $1,2,3,3,4,5]$

$$
M_{X(3872)}=\left\{\begin{array}{lc}
3875 \pm 0.7_{-2.0}^{+1.2} \mathrm{MeV} / \mathrm{c}^{2} & \text { Belle } \\
3871.8 \pm 3.1 \pm 3.0 \mathrm{MeV} / \mathrm{c}^{2} & \mathrm{D} 0 \\
3871.3 \pm 0.7 \pm 0.4 \mathrm{MeV} / \mathrm{c}^{2} & \mathrm{C} D F \\
3873.4 \pm 1.4 \mathrm{MeV} / \mathrm{c}^{2} & \text { BaBar } \\
3872.0 \pm 0.6 \pm 0.5 \mathrm{MeV} / \mathrm{c}^{2} & \text { Belle. }
\end{array}\right.
$$

The available experimental information indicates $J^{P C}=$ $1^{++}$for $X(3872)$ [6]. Theoretical interpretations of $X(3872)$ include a charmonium state [7], a molecular state 8], or the mixture of charmonium with molecular state 9$]$.

At present the observed decay modes of $X(3872)$ include $J / \psi \pi^{+} \pi^{-}$[1], $\gamma J / \psi$ [10], $J / \psi \pi^{+} \pi^{-} \pi^{0}$ [10] and $D^{0} \bar{D}^{0} \pi^{0}[5]$. The dipion in $J / \psi \pi^{+} \pi^{-}$seems to originate from $\rho \rightarrow \pi^{+} \pi^{-}$because the peak of the dipion invariant mass spectrum locates around $775 \mathrm{MeV} . J / \psi \pi^{+} \pi^{-} \pi^{0}$ comes from the sub-threshold decay $X(3872) \rightarrow J / \psi \omega$ [10]. Meanwhile the ratio of $B\left(X(3872) \rightarrow J / \psi \pi^{+} \pi^{-} \pi^{0}\right)$ to $B\left(X(3872) \rightarrow J / \psi \pi^{+} \pi^{-}\right)$given by experiment is $1.0 \pm 0.4$ (stat) \pm 0.3 (syst) [10]. Recently Belle experiment indicated $B\left(X(3872) \rightarrow D^{0} \bar{D}^{0} \pi^{0} K^{+}\right)=$ $9.4_{-4.3}^{+3.6} B\left(X(3872) \rightarrow J / \psi \pi^{+} \pi^{-} K^{+}\right)[5]$. Based on the above experimental data, one concludes (1) the $D^{0} \bar{D}^{* 0}$ is the dominant decay of $X(3872)$; (2) the isospin violating mode $X(3872) \rightarrow J / \psi \rho \rightarrow J / \psi \pi^{+} \pi^{-}$is not suppressed, compared with the isospin conserving mode $X(3872) \rightarrow J / \psi \omega \rightarrow J / \psi \pi^{+} \pi^{-} \pi^{0}$.

*Electronic address: xiangliu@pku.edu.cn
The Final State Interaction (FSI) effect sometimes plays a crucial role in many processes [11]. In this work, we study if the hidden charm decay $J / \psi \rho\left(\pi^{+} \pi^{-}\right)$mainly arises from the FSI effect of $X(3872) \rightarrow \bar{D}^{* 0} D^{0}+$ h.c.. $X(3872)$ decays to $D^{0} \bar{D}^{* 0}+$ h.c. but not $D^{+} D^{*-}+$ h.c. because $D^{0}+\bar{D}^{* 0}=3871.3 \mathrm{MeV}<M_{X(3872)}$ and $D^{+}+D^{*-}=3879.3 \mathrm{MeV}>M_{X(3872)}$. Thus the isospin violating process $X(3872) \rightarrow J / \psi \rho$ can occur via the $\bar{D}^{* 0} D^{0}+$ h.c. re-scattering effect.

This paper is organized as follows. We present the formulation about $X(3872) \rightarrow D^{0} \bar{D}^{* 0}+$ h.c. $\rightarrow J / \psi \rho$ in Section [II Then we present our numerical results. The last section is a short discussion.

\section{FORMALISM}

The Feynman diagrams for the $X(3872) \rightarrow J / \psi \rho$ through $\bar{D}^{* 0} D^{0}+$ h.c. re-scattering are depicted in Fig. 1.

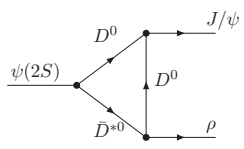

(a)

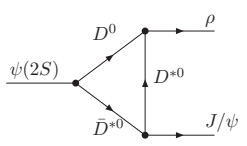

$(d)$

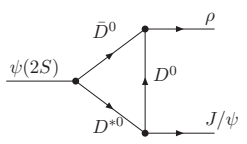

(g)

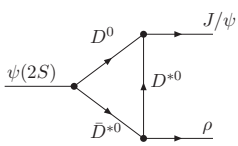

$(b)$
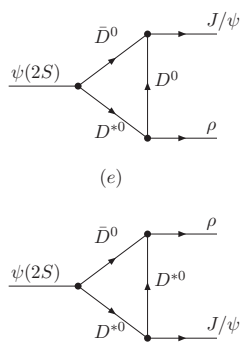

(h)

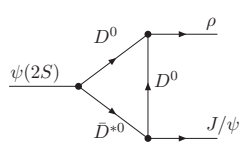

(c)

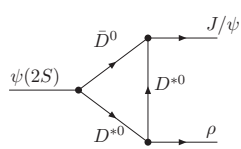

$(f)$
FIG. 1: The diagrams for $X(3872) \rightarrow \bar{D}^{* 0} D^{0}+$ h.c. $\rightarrow J / \psi \rho$. 
In Refs. [12, 13, 14], the effective Lagrangians, which are relevant to the present calculation, are constructed based on the chiral symmetry and heavy quark symmetry:

$$
\begin{aligned}
& \mathcal{L}=g_{X} X^{\mu}\left[D^{0} \bar{D}_{\mu}^{* 0}-\bar{D}^{0} D_{\mu}^{* 0}\right] \\
& +i g_{J / \psi \mathcal{D D}} \psi_{\mu}\left(\partial^{\mu} \mathcal{D} \mathcal{D}^{\dagger}-\mathcal{D} \partial^{\mu} \mathcal{D}^{\dagger}\right) \\
& -g_{J / \psi \mathcal{D}^{* \mathcal{D}}} \varepsilon^{\mu \nu \alpha \beta} \partial_{\mu} \psi_{\nu}\left(\partial_{\alpha} \mathcal{D}_{\beta}^{*} \mathcal{D}^{\dagger}+\mathcal{D} \partial_{\alpha} \mathcal{D}_{\beta}^{* \dagger}\right) \\
& -i g_{J / \psi \mathcal{D}^{*} \mathcal{D}^{*}}\left\{\psi^{\mu}\left(\partial_{\mu} \mathcal{D}^{* \nu} \mathcal{D}_{\nu}^{* \dagger}-\mathcal{D}^{* \nu} \partial_{\mu} \mathcal{D}_{\nu}^{* \dagger}\right)\right. \\
& +\left(\partial_{\mu} \psi_{\nu} \mathcal{D}^{* \nu}-\psi_{\nu} \partial_{\mu} \mathcal{D}^{* \nu}\right) \mathcal{D}^{* \mu \dagger}+\mathcal{D}^{* \mu}\left(\psi^{\nu} \partial_{\mu} \mathcal{D}_{\nu}^{* \dagger}\right. \\
& \left.\left.-\partial_{\mu} \psi_{\nu} \mathcal{D}^{* \nu \dagger}\right)\right\}+\left\{-i g_{\mathcal{D D V}} \mathcal{D}_{i}^{\dagger} \stackrel{\leftrightarrow}{\partial}{ }_{\mu} \mathcal{D}^{j}\left(\mathbb{V}^{\mu}\right)_{j}^{i}\right. \\
& -2 f_{\mathcal{D}^{*} \mathcal{D} \mathbb{V}} \varepsilon_{\mu \nu \alpha \beta}\left(\partial^{\mu} \mathbb{V}^{\nu}\right)_{j}^{i}\left(\mathcal{D}_{i}^{\dagger} \stackrel{\leftrightarrow}{\partial}^{\alpha} \mathcal{D}^{* \beta j}-\mathcal{D}_{i}^{* \beta \dagger} \stackrel{\leftrightarrow}{\partial} \mathcal{D}^{j}\right) \\
& +i g_{\mathcal{D}^{*} \mathcal{D}^{* V}} \mathcal{D}_{i}^{* \nu \dagger} \stackrel{\leftrightarrow}{\partial}_{\mu} \mathcal{D}^{* j}{ }_{\nu}\left(\mathbb{V}^{\mu}\right)_{j}^{i}
\end{aligned}
$$

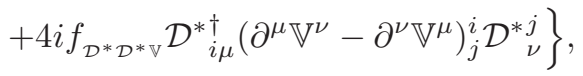

where $\mathcal{D}$ and $\mathcal{D}^{*}$ are the pseudoscalar and vector heavy mesons respectively, i.e. $\mathcal{D}^{(*)}=\left(\left(\bar{D}^{0}\right)^{(*)},\left(D^{-}\right)^{(*)}\right.$, $\left.\left(D_{s}^{-}\right)^{(*)}\right)$. The values of the coupling constants will be given in the following section. $\mathbb{V}$ denotes the nonet vector meson matrices

$$
\mathbb{V}=\left(\begin{array}{ccc}
\frac{\rho^{0}}{\sqrt{2}}+\frac{\omega}{\sqrt{2}} & \rho^{+} & K^{*+} \\
\rho^{-} & -\frac{\rho^{0}}{\sqrt{2}}+\frac{\omega}{\sqrt{2}} & K^{* 0} \\
K^{*-} & \bar{K}^{* 0} & \phi
\end{array}\right) .
$$

By the Cutkosky rule, the absorptive part of Fig. 1 (a) which comes from the re-scattering process of $X(3872) \rightarrow$ $D^{0}\left(p_{1}\right)+\bar{D}^{* 0}\left(p_{2}\right) \rightarrow J / \psi\left(p_{3}\right)+\rho\left(p_{4}\right)$ is written as

$$
\begin{aligned}
\operatorname{Abs}(a)= & \frac{1}{2} \int \frac{d^{3} p_{1}}{(2 \pi)^{3} 2 E_{1}} \frac{d^{3} p_{2}}{(2 \pi)^{3} 2 E_{2}} \\
& \times(2 \pi)^{4} \delta^{4}\left(m_{X}-p_{1}-p_{2}\right)\left[i g_{X} \varepsilon_{\xi}\right] \\
& \times\left[-g_{J / \psi D D} i\left(p_{1}-q\right) \cdot \varepsilon_{J / \psi}\right] \\
& \times\left[-\frac{2}{\sqrt{2}} i f_{D^{*} D V} \epsilon_{\mu \nu \alpha \beta} i p_{4}^{\mu} \varepsilon_{\rho}^{\nu}\left(i q^{\alpha}+i p_{2}^{\alpha}\right)\right] \\
& \times\left[-g^{\xi \beta}+\frac{p_{2}^{\xi} p_{2}^{\beta}}{m_{2}^{2}}\right]\left[\frac{i}{q^{2}-m_{D}^{2}}\right] \mathcal{F}^{2}\left(m_{D}, q^{2}\right) \\
= & \int d \Omega \frac{\left|\mathbf{p}_{1}\right|}{32 \pi^{2} m_{X}}\left[\sqrt{2} g_{X} g_{J / \psi D D} f_{D^{*} D V}\right] \\
& \times\left[\left(2 p_{1}-p_{3}\right) \cdot \varepsilon_{J / \psi}\right] \epsilon_{\mu \nu \alpha \beta} p_{4}^{\mu} \varepsilon_{\rho}^{\nu}\left(p_{3}^{\alpha}+p_{2}^{\alpha}-p_{1}^{\alpha}\right) \\
& \times\left[-\varepsilon^{\beta}+p_{2}^{\beta} \frac{p_{2} \cdot \varepsilon}{m_{2}^{2}}\right] \frac{\mathcal{F}^{2}\left(m_{D}, q^{2}\right)}{q^{2}-m_{D}^{2}}
\end{aligned}
$$

with $q^{2}=m_{1}^{2}+m_{3}^{2}-2 E_{1} E_{3}+2\left|\mathbf{p}_{1}\right|\left|\mathbf{p}_{3}\right| \cos \theta$, where $\mathcal{F}^{2}\left(m_{i}, q^{2}\right)$ etc denotes the form factors which compensate the off-shell effects of mesons at the vertices and are written as

$$
\mathcal{F}^{2}\left(m_{i}, q^{2}\right)=\left(\frac{\Lambda^{2}-m_{i}^{2}}{\Lambda^{2}-q^{2}}\right)^{2}
$$

where $\Lambda$ is a phenomenological parameter. As $q^{2} \rightarrow 0$ the form factor becomes a number. If $\Lambda \gg m_{i}$, it becomes unity. As $q^{2} \rightarrow \infty$, the form factor approaches to zero. As the distance becomes very small, the inner structure would manifest itself and the whole picture of hadron interaction is no longer valid. Hence the form factor vanishes and plays a role to cut off the end effect. The expression of $\Lambda$ is 15

$$
\Lambda\left(m_{i}\right)=m_{i}+\alpha \Lambda_{Q C D}
$$

where $m_{i}$ denotes the mass of exchanged meson. $\Lambda_{Q C D}=$ $220 \mathrm{MeV} . \alpha$ is a phenomenological parameter.

Similarly we obtain the absorptive contributions from Fig. 1 (b)-(d) respectively.

$$
\begin{aligned}
\operatorname{Abs}(b)= & \frac{1}{2} \int \frac{d^{3} p_{1}}{(2 \pi)^{3} 2 E_{1}} \frac{d^{3} p_{2}}{(2 \pi)^{3} 2 E_{2}} \\
& \times(2 \pi)^{4} \delta^{4}\left(m_{X}-p_{1}-p_{2}\right)\left[i g_{X} \varepsilon_{\xi}\right] \\
& \times\left[i g_{J / \psi D^{*}} \epsilon_{\mu \nu \kappa \sigma} \varepsilon_{J / \psi}^{\mu}(-i) p_{1}^{\nu}(-i) q^{\sigma}\right] \\
& \times\left\{-\frac{g_{D^{*} D^{*} V}}{\sqrt{2}} i\left(q+p_{2}\right) \cdot \epsilon_{\rho} g_{\alpha \beta}\right. \\
& \left.-\frac{4 f_{D^{*} D^{*} V}}{\sqrt{2}}\left[i p_{4 \beta} \epsilon_{\rho_{\alpha}}-i \epsilon_{\rho_{\beta}} p_{4 \alpha}\right]\right\} \\
& \times\left[-g^{\kappa \beta}+\frac{p_{2}^{\kappa} p_{2}^{\beta}}{m_{2}^{2}}\right]\left[-g^{\xi \alpha}+\frac{q^{\xi} q^{\alpha}}{m_{D^{*}}^{2}}\right] \\
& \times\left[\frac{i}{q^{2}-m_{D^{*}}^{2}}\right]\left(\frac{\Lambda^{2}-m_{D^{*}}^{2}}{\Lambda^{2}-q^{2}}\right)^{2},
\end{aligned}
$$

$$
\begin{aligned}
\operatorname{Abs}(c)= & \frac{1}{2} \int \frac{d^{3} p_{1}}{(2 \pi)^{3} 2 E_{1}} \frac{d^{3} p_{2}}{(2 \pi)^{3} 2 E_{2}} \\
& \times(2 \pi)^{4} \delta^{4}\left(m_{X}-p_{1}-p_{2}\right)\left[i g_{X} \varepsilon_{\xi}\right] \\
& \times\left[\frac{g_{D D V}}{\sqrt{2}} i\left(q-p_{1}\right) \cdot \varepsilon_{\rho}\right] \\
& \times\left[i g_{J / \psi D D^{*}} \epsilon_{\mu \nu \alpha \beta} \varepsilon_{J / \psi}^{\mu} i q^{\nu}(-i) p_{2}^{\beta}\right] \\
& \times\left[-g^{\xi \alpha}+\frac{p_{2}^{\xi} p_{2}^{\alpha}}{m_{2}^{2}}\right]\left[\frac{i}{q^{2}-m_{D}^{2}}\right]\left(\frac{\Lambda^{2}-m_{D}^{2}}{\Lambda^{2}-q^{2}}\right)^{2},
\end{aligned}
$$

$$
\begin{aligned}
\operatorname{Abs}(d)= & \frac{1}{2} \int \frac{d^{3} p_{1}}{(2 \pi)^{3} 2 E_{1}} \frac{d^{3} p_{2}}{(2 \pi)^{3} 2 E_{2}} \\
& \times(2 \pi)^{4} \delta^{4}\left(m_{X}-p_{1}-p_{2}\right)\left[i g_{X} \varepsilon_{\xi}\right] \\
& \times\left[-\frac{2}{\sqrt{2}} i f_{D^{*} D V} \epsilon_{\mu \nu \alpha \beta} i p_{3}^{\mu} \varepsilon_{\rho}^{\nu} i\left(q^{\alpha}-p_{1}^{\alpha}\right)\right] \\
& \times\left\{-g_{J / \psi D^{*} D^{*}}\left[i q^{\kappa} \varepsilon_{J / \psi}^{\sigma}+i p_{2}^{\sigma} \varepsilon_{J / \psi}^{\kappa}\right.\right. \\
& \left.\left.\times+i\left(p_{2}+q\right) \cdot \varepsilon_{J / \psi} g^{\kappa \sigma}\right]\right\}\left[-g_{\kappa}^{\xi}+\frac{p_{2_{\kappa}} p_{2}^{\xi}}{m_{2}^{2}}\right] \\
& \times\left[-g_{\sigma}^{\beta}+\frac{q_{\sigma} q^{\beta}}{m_{D^{*}}^{2}}\right]\left[\frac{i}{q^{2}-m_{D^{*}}^{2}}\right]\left(\frac{\Lambda^{2}-m_{D^{*}}^{2}}{\Lambda^{2}-q^{2}}\right)^{2} .
\end{aligned}
$$


The contributions from Fig. 1 (e), (f), (g), (h) is the same as that corresponding to Fig. 1 (a), (b), (c), (d) respectively.

The total amplitude of $X(3872) \rightarrow D^{0} \bar{D}^{* 0}+\bar{D}^{0} D^{* 0} \rightarrow$ $J / \psi \rho$ can be written as

$$
\mathcal{M}=2[\mathbf{A b s}(a)+\mathbf{A} \mathbf{b s}(b)+\mathbf{A} \mathbf{b s}(c)+\mathbf{A} \mathbf{b s}(d)],(10)
$$

where the pre-factor "2" comes from the consideration that the contribution from $D^{0} \bar{D}^{* 0}$ re-scattering is the same as that from $\bar{D}^{0} D^{* 0}$ re-scattering.

Because the $\rho$ meson is a broad resonance with $\Gamma_{\rho} \sim$ $150 \mathrm{MeV}$, the decay width of $X(3872) \rightarrow D^{0} \bar{D}^{* 0}+$ $\bar{D}^{0} D^{* 0} \rightarrow J / \psi \rho$ is written as

$\Gamma=\int_{0}^{\left(M_{X(3872)}-m_{J / \psi}\right)^{2}} \mathrm{~d} s f\left(s, m_{\rho}, \Gamma_{\rho}\right) \frac{|\mathbf{k}|\left|\mathcal{M}\left(m_{\rho} \rightarrow \sqrt{s}\right)\right|^{2}}{24 \pi M_{X(3872)}^{2}}$,

where the Breit-Wigner distribution function $f\left(s, m_{\rho}, \Gamma_{\rho}\right)$ and the decay momentum $|\mathbf{k}|$ are

$$
\begin{gathered}
f\left(s, m_{\rho}, \Gamma_{\rho}\right)=\frac{1}{\pi} \frac{m_{\rho} \Gamma_{\rho}}{\left(s-m_{\rho}^{2}\right)^{2}+m_{\rho}^{2} \Gamma_{\rho}^{2}}, \\
|\mathbf{k}|=\frac{\sqrt{\left[M_{X(3872)}^{2}-\left(\sqrt{s}+m_{J / \psi}\right)^{2}\right]\left[M_{X(3872)}^{2}-\left(\sqrt{s}-m_{J / \psi}\right)^{2}\right]}}{2 M_{X(3872)}} .
\end{gathered}
$$

\section{NUMERICAL RESULTS}

The coupling constants related to our calculation include 15]:

$$
\begin{aligned}
g_{D D V} & =g_{D^{*} D^{*} V}=\frac{\beta g_{V}}{\sqrt{2}}, \quad f_{D^{*} D V}=\frac{f_{D^{*} D^{*} V}}{m_{D^{*}}}=\frac{\lambda g_{V}}{\sqrt{2}} \\
g_{V} & =\frac{m_{\rho}}{f_{\pi}}
\end{aligned}
$$

where $f_{\pi}=132 \mathrm{MeV}, g_{V}, \beta$ and $\lambda$ are parameters in the effective chiral Lagrangian that describes the interaction of heavy mesons with the low-momentum light vector mesons [14]. Following Ref. [16], we take $g=0.59$, $\beta=0.9$ and $\lambda=0.56$. Based on the vector meson dominance model and using $J / \psi$ 's leptonic width, the authors of Ref. [17] determined $g_{J / \psi \mathcal{D} \mathcal{D}}^{2} /(4 \pi)=5$. As a consequence of the spin symmetry in the heavy quark effective field theory, $g_{J / \psi \mathcal{D D}^{*}}$ and $g_{J / \psi \mathcal{D}^{*} \mathcal{D}^{*}}$ satisfy the relations: $g_{J / \psi \mathcal{D} \mathcal{D}^{*}}=g_{J / \psi \mathcal{D} \mathcal{D}} / m_{D}$ and $g_{J / \psi \mathcal{D}^{*} \mathcal{D}^{*}}=g_{J / \psi \mathcal{D} \mathcal{D}}$ [18].

By fitting the upper limit of the total width of $X(3872)$ $(2.3 \mathrm{MeV})$, one obtains the coupling constant $g_{X}$ in Eq. (2)

$$
g_{X}=\left\{\begin{array}{l}
2.2 \mathrm{GeV}, \text { for } M_{X(3872)}=3875.0 \mathrm{MeV} \\
2.5 \mathrm{GeV}, \text { for } M_{X(3872)}=3873.4 \mathrm{MeV} \\
3.1 \mathrm{GeV}, \text { for } M_{X(3872)}=3872.0 \mathrm{MeV}
\end{array}\right.
$$

where we approximately take $\bar{D}^{* 0} D^{0}$ as the dominant decay mode of $X(3872)$ considering the experimental result [5]: $B\left(X(3872) \rightarrow D^{0} \bar{D}^{0} \pi^{0} K^{+}\right)=9.4_{-4.3}^{+3.6} B(X(3872) \rightarrow$ $\left.J / \psi \pi^{+} \pi^{-} K^{+}\right)$.

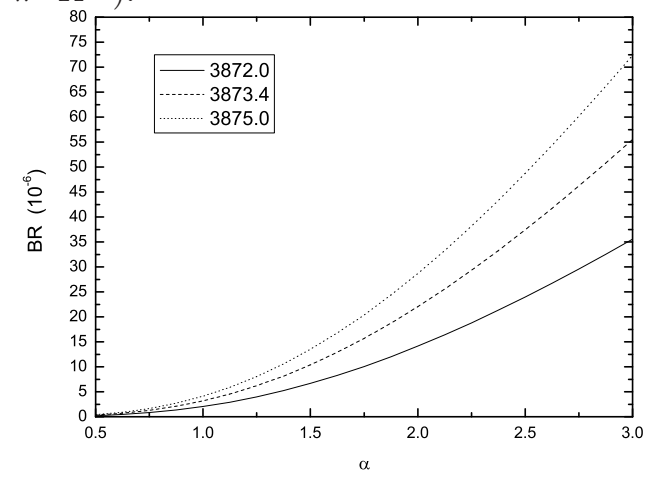

FIG. 2: The dependence of decay width of $X(3872) \rightarrow$ $D^{0} \bar{D}^{* 0}+$ h.c. $\rightarrow J / \psi \rho$ on $\alpha$.

The value of $\alpha$ in the form factor usually is of order unity [15]. In this work we take the range of $\alpha=0.5 \sim 3$. The dependence of the branching ratio of $X(3872) \rightarrow$

\begin{tabular}{|c|c|c|c|c|c|c|}
\hline $\operatorname{Mass}(\mathrm{MeV}) \quad \alpha$ & 0.5 & 1.0 & 1.5 & 2.0 & 2.5 & 3.0 \\
\hline $3872.0[1]$ & $2.1 \times 10^{-7}$ & $2.0 \times 10^{-6}$ & $6.6 \times 10^{-6}$ & $1.4 \times 10^{-5}$ & $2.4 \times 10^{-5}$ & $3.6 \times 10^{-5}$ \\
\hline $3873.4[2]$ & $3.2 \times 10^{-7}$ & $3.2 \times 10^{-6}$ & $1.0 \times 10^{-5}$ & $2.2 \times 10^{-5}$ & $3.7 \times 10^{-5}$ & $5.6 \times 10^{-5}$ \\
\hline $3875.0[5]$ & $4.2 \times 10^{-7}$ & $4.1 \times 10^{-6}$ & $1.3 \times 10^{-5}$ & $2.9 \times 10^{-5}$ & $4.9 \times 10^{-5}$ & $7.2 \times 10^{-5}$ \\
\hline
\end{tabular}
$D^{0} \bar{D}^{* 0} \rightarrow J / \psi \rho$ on $\alpha$ is presented in Fig. 2 .

In Table I we list the typical values of the branching ratio of $X(3872) \rightarrow D^{0} \bar{D}^{* 0}+$ h.c. $\rightarrow J / \psi \rho$ when we take several masses of $X(3872)$ from various experiments and different $\alpha$.

TABLE I: The typical values of branching ratio of $X(3872) \rightarrow D^{0} \bar{D}^{* 0}+$ h.c. $\rightarrow J / \psi \rho$ for different values of $M_{X(3872)}$ and $\alpha$.

\section{DISCUSSION}

Understanding the large $J / \psi \rho$ decay width of $X(3872)$ may help reveal the nature of $X(3872)$. In this work, we study if the large branching ratio of $X(3872) \rightarrow J / \psi \rho$ 


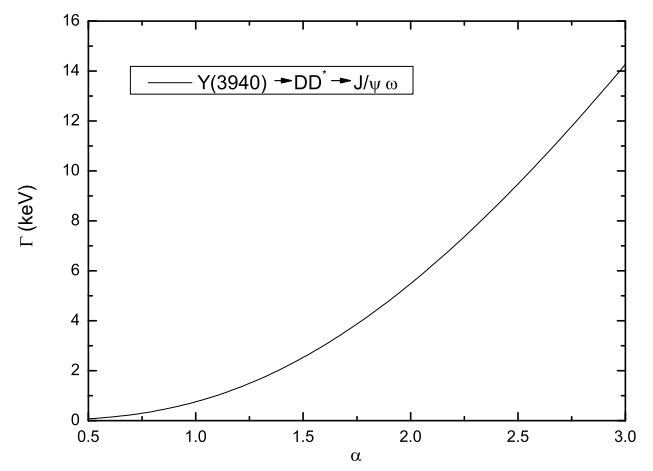

FIG. 3: The dependence of the decay width of $Y(3940) \rightarrow$ $D \bar{D}^{*} \rightarrow J / \psi \omega$ on $\alpha$.

can be explained by the $X(3872) \rightarrow D^{0} \bar{D}^{0 *}+$ h.c. rescattering effect. The numerical results indicate that $B\left(X(3872) \rightarrow D^{0} \bar{D}^{0 *}+\right.$ h.c. $\left.\rightarrow J / \psi \rho\right)$ is about $10^{-5} \sim$ $10^{-7}$. Thus the large isospin violating $X(3872) \rightarrow J / \psi \rho$ decay width can hardly be attributed to the FSI effect of $X(3872) \rightarrow D^{0} \bar{D}^{0 *}+$ h.c.. The suppression from the phase space of $X(3872) \rightarrow D^{0} \bar{D}^{0 *}+$ h.c. is huge because the experimental values of $X(3872)$ mass is only barely above the $J / \psi+\rho$ or $D^{* 0}+D^{0}$ threshold, although the re-scattering effect is obvious.

The reliable dynamical calculation of the hidden charm decay width has been a challenging theoretical issue for decades. In Ref. [19], the explicit $J / \psi \rho$ component is introduced into the $X(3872)$ wave function in order to explain the large $J / \psi \pi \pi$ decay width of $X(3872)$. In Ref. [20], QCD multipole expansion technique was used to calculate hadronic transitions such as $\psi(2 S) \rightarrow J / \psi \pi \pi$ and $\Upsilon(n s) \rightarrow \Upsilon(1 s) \pi \pi$. If the main component of $X(3872)$ is $c \bar{c}$ [9, 21], the large $X(3872) \rightarrow J / \psi \pi \pi$ decay might also be understood with this approach.

Last year, Belle reported a new charmonium state $Y(3940)$ in the channel $B \rightarrow J / \psi \omega K$ and obtained $B(B \rightarrow Y(3940)+K) \cdot B(Y(3940) \rightarrow \omega J / \psi)=(7.1 \pm$ $1.3 \pm 3.1) \times 10^{-5}$. Its mass and width are $3946 \pm 11$ (stat) \pm 13 (syst) $\mathrm{MeV}$ and $87 \pm 22($ stat $) \pm 26$ (syst) $\mathrm{MeV}[22]$ respectively. In particular, $\Gamma[Y(3940) \rightarrow J / \psi \omega]>7 \mathrm{MeV}$. Godfrey suggested $Y(3940)$ as the $\chi_{c 1}^{\prime}$ state with quantum number $2^{3} P_{1}$ [23] and indicated that $Y(3940) \rightarrow$ $J / \psi \omega$ might come from the FSI effect of $Y(3940) \rightarrow$ $D \bar{D}^{*}+$ h.c.. In Fig. 3] we present the dependence of the width of $Y(3940) \rightarrow D \bar{D}^{*}+$ h.c. $\rightarrow J / \psi \omega$ on $\alpha[24$, where one takes $D \bar{D}^{*}$ as the dominant decay mode of $Y(3940)$ as suggested in Ref. [23]. The order of magnitude of $\Gamma\left[Y(3940) \rightarrow D \bar{D}^{*}+\right.$ h.c. $\left.\rightarrow J / \psi \omega\right]$ is keV, which is far less than Belle's data. Clearly more experimental information on $Y(3940)$ will be very helpful.

\section{Acknowledgments}

S.L.Z thanks Prof. K.T. Chao for helpful discussions. This project was supported by the National Natural Science Foundation of China under Grants 10375003, 10421503 and 10625521, Key Grant Project of Chinese Ministry of Education (No. 305001).
[1] S.K. Choi et al., Belle Collaboraion, Phys. Rev. Lett. 91, 262001 (2003).

[2] B. Aubert et al., Babar Collaboration, Phys. Rev. D 71, 071103 (2005).

[3] D. Acosta et al., CDFII Collaboration, Phys. Rev. Lett. 93, 072001 (2004).

[4] V.M. Abazov et al., D0 Collaboration, Phys. Rev. Lett. 93, 162002 (2004).

[5] G. Gokhroo et al., Belle Collaboration, arXiv: hep-ex/0606055.

[6] K. Abe et al., Belle Collaboration, arXiv: hep-ex/0505038.

[7] T. Barnes, S. Godfrey and E.S. Swanson, Phys. Rev. D 72, 054026 (2005); E.J. Eichten, K. Lane and C. Quigg, Phys. Rev. D 73, 014014 (2006); T. Barnes and S. Godfrey, Phys. Rev. D 69, 054008 (2004); E.J. Eichten, K. Lane and C. Quigg, Phys. Rev. D 69, 094019 (2004).

[8] F.E. Close and P. Page, Phys. Lett. B 579, 67 (2004); N.A. Tornqvist, Phys. Lett. B 590, 209 (2004); C.Y. Voloshin, Phys. Lett. B 579, 316 (2004); C.Y. Wong, Phys. Rev. C 69, 055202 (2004); E. Braaten and M. Kusunoki, Phys. Rev. D 69, 074005 (2004); E.S. Swanson, Phys. Lett. B 588, 189 (2004); E.S. Swanson, Phys. Lett. B 598, 197 (2004).

[9] C. Meng, Y.J. Gao and K.T. Chao, arXiv: hep-ph/0506222

[10] K. Abe et al., Belle Collaboration, arXiv: hep-ex/0505037.

[11] N. Isgur, K. Maltman, I. Weinstein and T. Barnes, Phys. Rev. Lett. 64, 161 (1990); M.P. Locher, V.E. Markusin and H.Q. Zheng, Report No. PSI-PR-96-13 (unpublished); H. Lipkin, Nucl. Phys. B 244, 147 (1984); H. Lipkin, Phys. Lett. B 179, 278 (1986); H. Lipkin, Nucl. Phys. B 291, 720 (1987); H. Lipkin and B.S. Zou, Phys. Rev. D 53, 6693 (1996); P. Geiger and N. Isgur, Phys. Rev. Lett. 67, 1066 (1991); V.V. Anisovich, D.V. Bugg, A.V. Sarantsev and B.S. Zou, Phys. Rev. D 51, R4619 (1995); X.Q. Li, D.V. Bugg and B.S. Zou, Phys. Rev. D 55, 1421 (1997).

[12] Y. Oh, T. Song and S.H. Lee, Phys. Rev. C 63, 034901 (2001).

[13] H.Y. Cheng, C.Y. Cheung, G.L. Lin, Y.C. Lin, T.M. Yan and H.L. Yu, Phys. Rev. D 47, 1030 (1993); T.M. Yan, H.Y. Cheng, C.Y. Cheung, G.L. Lin, Y.C. Lin and H.L. Yu, Phys. Rev. D 46, 1148 (1992). M. B. Wise, Phys. Rev. D 45, R2188 (1992); G. Burdman and J. Donoghue, Phys. Lett. B 280, 287 (1992).

[14] R. Casalbuoni, A. Deandrea, N. Di Bartolomeo, R. Gatto, F. Feruglio and G. Nardulli, Phys. Rep. 281, 145 (1997). 
[15] H.Y. Cheng, C.K. Chua and A. Soni, Phys. Rev. D71, 014030 (2005).

[16] C. Isola, M. Ladisa, G. Nardulli and P. Santorelli, Phys. Rev. D 68, 114001 (2003).

[17] N.N. Achasov and A.A. Kozhevnikov, Phys. Rev. D 49, 275 (1994).

[18] A. Deandrea, G. Nardulli and A.D. Polosa, Phys. Rev. D 68, 034002 (2003).

[19] E.S. Swanson, Phys. Lett. B 588, 189 (2004).

[20] T.M. Yan, Phys. Rev. D 22, 1652 (1980); Y.P. Kuang, Front. Phys. China 1, 19 (2006).
[21] M. Suzuki, Phys. Rev. D 72, 114013 (2005).

[22] S.K. Choi, Belle Collaboration, Phys. Rev. Lett. 94, $182002(2005)$.

[23] S. Godfrey, eConf C060409: 015, 2006. arXiv: hep-ph/0605152

[24] For the calculation of $Y(3940) \rightarrow D \bar{D}^{*} \rightarrow J / \psi \omega$, we only replace relevant masses and coupling constant in the formulas of $X(3872) \rightarrow D^{0} \bar{D}^{* 0}+$ h.c. $\rightarrow J / \psi \rho$ to make a rough estimate. Other intermediate mesons for $Y(3940) \rightarrow J / \psi \omega$ are also allowed. 